\title{
Nondimeno. \\ Una nota sul linguaggio della circostanza e dell'eccezione nel Carteggio sforzesco
}

\author{
di Massimo Della Misericordia
}

Sulla scorta della recente monografia di C. Ginzburg e dei rilievi di Pedullà, è possibile identificare nella parola nondimanco/nondimeno un passaggio importante della scrittura rinascimentale del politico. Essa però non compare solo nelle opere di Machiavelli o Guicciardini e nella riflessione intellettualmente più consapevole, ma anche nell'ingente mole di lettere che costituiscono i carteggi di governo del tempo. In questo tipo di testi pragmatici conservatisi per lo stato di Milano in età sforzesca ricorre come una parola-chiave dell'attrito fra legge e trasgressione (o eccezione ritenuta legittima), fra diritto e pratica. Rivela il dilemma tra un assunto investito di valore (il duca deve onorare i contenuti dei capitoli convenuti con i sudditi e le sue promesse, la consuetudine esige rispetto, le divisioni fazionarie vanno superate) e il timore che esso venisse calpestato o invece la volontà, se non la necessità, di attenuarlo. Questa congiunzione sintetizzò così l'esigenza di sfumare la norma, adeguarla alla circostanza, far convivere diritti o ragioni di opportunità potenzialmente contrastanti. Si pone insomma come spia di uno dei principali motivi di tensione scoperti nello stato tardo-medievale, dibattuti da una lunga tradizione di studi che va da O. Brunner a R. Fubini, fra l'autorità del principe, quale arbitro dell'eccezione richiesta di volta in volta dalla stessa varietà delle situazioni concrete, e la cultura legalitaria dei corpi territoriali, i quali richiamandosi al diritto e alla consuetudine cercarono di arginare i poteri «straordinari» che il signore veniva attribuendosi.

On the basis of the recent monograph by C. Ginzburg and G. Pedullà's review, it is possible to identify the word nondimanco/nondimeno (nonetheless) as an important element in Renaissance political writing. However, it does not only appear in the work of Machiavelli or Guicciardini and in the more conscious reflections by the intellectuals, but also in the huge amount of letters that constitute the government correspondence of the time. In these kinds of pragmatic texts, referring to the state of Milan in the Sforza age, it recurs as a key word of a dilemma: the friction between law and transgression (or exception considered legitimate) and also between law and practice. On one hand, it expresses an assumption invested in value: the duke must hon-

\footnotetext{
Massimo Della Misericordia, University of Milano-Bicocca, Italy, massimo.dellamisericordia@unimib.it, 0000-0002-4564-0277

FUP Best Practice in Scholarly Publishing (DOI 10.36253/fup_best_practice)

Massimo Della Misericordia, Nondimeno. Una nota sul linguaggio della circostanza e dell'eccezione nel Carteggio sforzesco, pp. 95-110, (C) 2021 Author(s), content CC BY 4.0 International, DOI 10.36253/978-885518-423-6.06, in Paola Guglielmotti, Isabella Lazzarini (edited by), «Fiere vicende dell'età di mezzo». Studi per Gian Maria Varanini, (c) 2021 Author(s), content CC BY 4.0 International, metadata CC0 1.0 Universal, published by Firenze University Press (www.fupress.com), ISSN 2704-6079 (online), ISBN 978-88-5518423-6 (PDF), DOI 10.36253/978-88-5518-423-6
} 
or his promises and the contents of the chapters agreed on with his subjects; custom demands respect; factional divisions must be overcome. At the same time it reveals the concern that this principle could be trampled upon, or instead the will, if not the need, to attenuate the more general rule. This conjunction thus summarized the requirement to nuance the law, to adapt it to circumstance, and to conciliate potentially conflicting rights or reasons. In short, it stands as an indicator of one of the main causes of open tensions in the late medieval state, debated by a long tradition of scholars ranging from O. Brunner to R. Fubini: the opposition between the authority of the prince, as arbiter of the exception requested from time to time from the same variety of concrete situations, and the legalistic culture of the territorial bodies, which, referring to law and custom, tempted to stem the "extraordinary" powers that the duke was attributing to himself.

Tardo medioevo; secoli XX-XXI; storia della storiografia; Carteggio sforzesco; linguaggi politici; legittimità del principe eccezione.

Later Middle Ages; $2 \mathrm{O}^{\text {th }}-21^{\text {st }}$ centuries; historiography; Carteggio sforzesco; political languages; prince legitimacy; exception.

\section{Scritture rinascimentali del politico}

Carlo Ginzburg, nel libro che segue i sentieri che collegano o separano Machiavelli, la casistica, ulteriore oggetto dell'interesse recente dell'autore, Pascal, i gesuiti, Galileo e altri momenti di snodo della cultura europea, conferisce un ruolo chiave alla ricorrenza della parola nondimanco nelle pagine del Principe, dei Discorsi, ma anche delle Legazioni e commissarie, al punto da farne il titolo dell'opera. La congiunzione svelerebbe la tensione fra il generale e il fatto particolare, fra il principio e la deroga, tema centrale della formazione di Machiavelli, che «imparò dalla casistica medievale a riflettere sulla norma e sull'eccezione» (p. 11), della sua opera artistica e della sua riflessione teorica. Nella raccolta di saggi, peraltro, emerge chiaramente l'acuta coscienza, in Machiavelli, degli incessanti scarti della realtà rispetto ai tentativi di regolarizzazione, insieme al convinto apprezzamento dell'esperienza, che ci introducono all'insieme di problemi che qui affronteremo, quelli posti ad ogni regime politico dalla necessità di «adattarsi ad un mondo mutevole» (p. 58)1.

Fra le letture del libro si segnala, per ampiezza e analiticità, quella di Gabriele Pedullà. Il recensore si esprime criticamente sulle interpretazioni centrali di Ginzburg, essenzialmente il rapporto di Machiavelli con l'aristotelismo, con la casistica e la tradizione giuridica ${ }^{2}$. D’altra parte anche il lavoro di Pedullà esprime uno sforzo analogo di situare i problemi intellettuali di Machiavelli in luoghi lontani non solo dagli stereotipi polemici, ma anche dalle tradizionali dicotomie (espresse in termini sovente anacronistici) principato/ repubblicanesimo, ragion di stato/bene comune, politica/morale. Semmai si ridisegna profondamente il quadro delle influenze e del contesto in cui mi-

${ }^{1}$ Ginzburg, Nondimanco. Per ragioni di spazio, dei vari autori è di prassi richiamato un solo titolo di riferimento.

${ }^{2}$ Su questa materia, si veda Quaglioni, Machiavelli, pp. 62-76. 
surare l'originalità del Principe e dei Discorsi. Pedullà, infatti, segnala come la derogabilità della norma fosse già «profondamente inscritta» nel diritto romano (p. 22). Riconosce l'interesse di Machiavelli per il tema dell'eccezione, ma in un più ampio dialogo quattrocentesco e poi cinquecentesco, che sollecita un confronto tra figure intellettuali, eventualmente anche quelle considerate minori (p. 68). Pone, infine, le questioni dell'esperienza della «politica vissuta» (p. 64) dal segretario fiorentino e di una puntuale considerazione della politica raccontata, vale a dire le «legazioni, commissarie e scritti di governo dello stesso Machiavelli», ma anche le consulte e pratiche, i vari materiali cancellereschi, utili «per impratichirsi della lingua del Principe e dei Discorsi» (pp. 82-83) . $^{3}$

Ora, sia Ginzburg sia Pedullà, entro una produzione di studi machiavelliani sempre copiosa, si sono mostrati interessati soprattutto a discutere, e a prendere le distanze, dal Machiavelli della cosiddetta Scuola di Cambridge, riletto alla luce delle virtù civiche da storici del pensiero politico come John G.A. Pocock e Quentin Skinner. È invece rimasto più sullo sfondo il contributo degli storici proprio di quella politica vissuta, così evocata prima, che pure hanno guardato a Machiavelli come a un testimone, uno storico a sua volta, un critico o uno specchio delle prassi di governo quattro-cinquecentesche. In questo senso sono intervenuti molti studiosi e studiose, e fra quelli che hanno lasciato una traccia più profonda, dei regimi del tempo. Anche un cursorio richiamo consente di percepire i profondi mutamenti degli interessi storiografici, che sono mutamenti di più generali orientamenti culturali, sino ad una significativa convergenza, anche in questo caso, con i grandi temi della filosofia contemporanea che, si pensi solo a Jacques Derrida e Giorgio Agamben, ha fatto dell'eccezione una prospettiva privilegiata per l'analisi e la decostruzione del politico.

In una fase di adesione ancora solida all'evoluzione della modernità, si poteva leggere in Machiavelli «la fede appassionata nello stato forte e sano», pure in un quadro di drammatica "crisi" nazionale. Così scriveva Federico Chabod, molte delle cui pagine, pure riproposte a suggello di un'antologia di notevole impatto degli anni Settanta, hanno perso attualità. Restano però suscettibili di approfondimento, anche in questa sede, la sfumata caratterizzazione dei poteri principeschi, con la novità della plenitudo potestatis pretesa dai signori e, d'altra parte, l'incertezza della tradizione politica di quei regimi, avversata dalla tenace cultura giuridica e consuetudinaria dei corpi territoriali ${ }^{4}$. Gli studiosi delle generazioni successive hanno problematizzato attraverso Machiavelli i temi venuti alla ribalta dagli anni Sessanta, come quelli del mutamento politico che dalle democrazie comunali condusse alle serrate oligarchiche e ai principati, dunque la flessione della rappresentanza, le clientele e le divisioni interne, nonché il superamento del quadro della città-stato

3 Pedullà, Machiavelli secondo Carlo Ginzburg.

4 Chabod, La genesi del «Principe», p. 324 per la citazione. 
nelle nuove gerarchie territoriali fra le dominanti e i centri soggetti ${ }^{5}$. Gli interrogativi del mio intervento sono però in sintonia soprattutto con le ricerche di Riccardo Fubini, sebbene io ritenga problematica, sul piano generale, la sua propensione a leggere questi aspetti della politica rinascimentale nella proiezione della modernità. Per dare «un attendibile quadro di riferimento al pensiero di Machiavelli» (p. 275), egli ha ritenuto di approfondire le riflessioni che accompagnarono la rottura costituzionale, la cui radicalità è già un sentito tema burckhardtiano, rappresentata dai regimi affermatisi in Italia nel temporaneo appannamento dei «poteri universali e regolativi di Chiesa ed Impero» (p. 8), con i loro stili di governo di fatto «in deroga (...) ad assodate tradizioni e garanzie dei cittadini» (p. 281). La concretezza di una vita politica difficile da racchiudere nell'ordine legale di matrice comunale favorì la ricerca, oltre la tradizione teologica e giuridica, degli strumenti concettuale utili a fronteggiare una «sfuggente realtà» (p. 275), che a stento poteva essere ingabbiata nella regolarizzazione normativa o nelle convenzioni intellettuali del passato, sottraendo la sua «novità» (p. 285) alla connotazione negativa che gravava su questo concetto. Torniamo così al nostro tema delle «eccezioni alla legge» (p. 9), allo «stato di eccezionalità» (p. 11), espressione con cui Fubini traduce quella coeva, molto familiare a Machiavelli, di «modi straordinari», cui ricorrevano repubbliche e principati con il conferimento e l'esercizio di poteri speciali.

Tutti gli storici citati hanno mostrato interesse per il linguaggio e lo stile di Machiavelli. Fubini situa Machiavelli in una «culla» costituita fra l'altro dai testi di celebri umanisti, da una spregiudicata «produzione epistolare privata o politico-diplomatica» (p. 282), dagli «ambienti politico-cancellereschi» e dalla testualità che vi veniva prodotta (p. 283 $)^{6}$. Pedullà segnala l'uso della stessa parola nondimanco fra altri scrittori del Quattrocento ${ }^{7}$. Queste pagine sono dedicate appunto alla coeva circolazione dei linguaggi di sottile sfumatura della norma e in particolare della congiunzione dell'eccezione nelle cancellerie $o$ in sedi più periferiche della scrittura della prassi politica. «Niente de meno» è registrato dal brillante diplomatico e ufficiale sforzesco Nicodemo Tranchedini da Pontremoli nel suo vocabolario della lingua volgare ${ }^{8}$. Nondimanco o, come del resto in Machiavelli, nondimeno o niente de meno o de manco, con ulteriori varianti grafiche, torna spesso in uno dei più ricchi car-

5 Fasano Guarini, Repubbliche e principi; The Cambridge Companion to Machiavelli; Connell, Machiavelli nel Rinascimento.

${ }^{6}$ Fubini, Politica e pensiero.

7 Pedullà, Machiavelli secondo Carlo Ginzburg, p. 20. Si veda anche la puntualizzazione della replica (Ginzburg, Gabriele Pedullà, p. 194), che a mio avviso ripropone l'esigenza di identificare un «contesto» non «generico» per questo linguaggio, come qui ci si ripropone di fare con le scritture pragmatiche del politico. L'uso frequente di nondimeno/non di mancho da parte di Francesco Guicciardini, nel carteggio, nelle opere teoriche e storiche, per esprimere ardue compatibilità ad esempio fra il diritto e l'opportunità o fra le strutture costituzionali e la realtà politica, emerge da La Storia d'Italia, passim, e Baja Guarienti, Il bandito e il governatore, p. 69.

8 Tranchedini, Vocabolario italiano-latino, p. 114. 
teggi prodotti dalle relazioni di potere nel XV secolo, la massiccia mole di carte conservatasi per lo stato di Milano, offrendoci la materia di un ulteriore approfondimento.

\section{Regole e deroghe nel linguaggio del Carteggio sforzesco}

Non si vuole sovraccaricare di significato una ricorrenza prevedibile e molto spesso piatta, in casi che non vale la pena enumerare. Spesso la congiunzione oppone, in modo semplice, il buon diritto e la difficoltà di affermarlo oppure uno statuto, un decreto o una grida e la relativa infrazione. Eppure, considerate le sue potenzialità, di esprimere cioè la doppia validità di due affermazioni parzialmente contrastanti, una delle quali però limita la portata dell'altra, graduando elasticamente ragioni personali o istituzionali, un sondaggio senza ambizioni di esaustività consente di rinvenirla in passaggi emblematici, che richiedevano una sottile articolazione del discorso politico' ${ }^{\text {. }}$ Non si tratta propriamente della circostanza in cui venga enunciata una regolarità composta di un principio di validità più estesa e dell'anomalia, come, diciamo così, nella politologia machiavelliana (per fare l'esempio di una questione spinosa anche nei casi che presenteremo: «quanto sia laudabile in uno principe mantenere la fede (...), ciascuno lo intende; nondimanco si vede, per esperienzia ne' nostri tempi quelli principi avere fatto grandi cose, che della fede hanno tenuto poco conto») ${ }^{10}$. Incontriamo in ogni caso significativi attriti fra legge e trasgressione (o eccezione ritenuta legittima), fra diritto e pratica, fra un assunto investito di valore (il duca deve onorare i contenuti dei capitoli convenuti con i sudditi e le sue promesse, la consuetudine esige rispetto, le divisioni parziali vanno superate) e il timore che esso venga calpestato o al contrario la volontà, quando non la necessità, di attenuarlo. La congiunzione è inoltre un indicatore di quella complessità sintattica di cui è stata offerta anche un'interpretazione politica: crescente nelle scritture volgari della prassi nel XV secolo, alimentata senz'altro da fenomeni squisitamente culturali (come la dimestichezza con i classici), per altro verso accompagna intrinsecamente una specifica narrazione, e comprensione, delle relazioni diplomatiche, cioè delle sfaccettature dei loro problemi e delle mediazioni da ricercare con reciproche concessioni ${ }^{11}$.

La sfuggente complessità della politica consisteva, fra l'altro, nellirriducibilità della competizione e delle alleanze di partito a schemi semplici. A Parma si stabilizzò una divisione fra quattro squadre, fazioni che facevano capo a quattro famiglie, mediante la quale si organizzarono la ripartizione dei

9 Per avere il senso della frequenza del termine, basti considerare la monografia, dedicata al conflitto politico e al suo linguaggio, di Leprai, Il governo del disordine, pp. 197, 213, nota 12, p. 227, nota 54, p. 256.

${ }_{10} \mathrm{E}$ uno dei passi messi in risalto da Ginzburg, Nondimanco, p. 28.

${ }^{11}$ Lazzarini, Argument and emotion, pp. 355-356. 
seggi consiliari e delle altre cariche, le relazioni fra clienti e patroni, come fra città e contado. Quando l'equilibrio si alterò ne derivarono momenti di grave instabilità politica. Il quadro però era più complesso di questa formalizzazione e Antonio Colli cercò di decifrarlo. Il legumdoctor di origine vigevanese e di formazione pavese, che ricoprì molti uffici periferici e divenne vicario generale, influente carica centrale, intorno al 1470 fu vicario del podestà nella città emiliana. Indirizzò allora al duca una lettera che illustrava il sistema, insistendo però soprattutto sulle divisioni all'interno delle squadre e sulle alleanze trasversali rispetto alle squadre stesse (molti esponenti delle diverse squadre erano conligati tra loro). Di più, pareva all'ufficiale di grande importanza soprattutto la distinzione fra $\mathrm{i}$ «fideli servitori» dello stato e quelli che non lo erano, che a sua volta attraversava le squadre. Riporto un passaggio rilevante di questo sforzo di argomentare fra il livello istituzionale, i dati di una realtà meno evidente e un'ulteriore eccezione. «Non obstante che essa cità sia divisa per quatro squadre, cioè ducali, rossa, palavicina e sanvidali, nondemancho sono esse squadre excepto la rossa tanto divisi tra loro che certo è un gran fato» ${ }^{12}$.

Analogamente la distanza fra la norma giuridica e la varietà delle situazioni reali, nella vita di tutti i giorni non meno che nella vita pubblica, imponeva mediazioni non imprigionate dal formalismo. Si possono prendere le mosse da una circostanza di scarso peso politico, ma che tocca una figura che ci conduce al cuore di quella cultura cancelleresca cui si riferivano Pedullà e Fubini, Cicco Simonetta. Al servizio di Francesco Sforza, divenne il primo segretario dello stato di Milano e dopo l'assassinio di Galeazzo Maria esercitò uno strapotere che acuì l'odio della nobiltà milanese, portandolo alla rovina $\mathrm{e}$ al patibolo. Nel 1471 il Simonetta era in lite con Pietro da Gallarate per l'uso irriguo delle acque del Sesia. Nella carta scritta, per suo conto, dal fedelissimo Giacomo Griffo, podestà di Sartirana, egli manifestava il convincimento di avere tutte le ragioni: quelle della consuetudine, dei privilegi ducali e di una sentenza della giustizia pavese. Nientedimeno proponeva un compromesso amichevole, per affezione scriveva, invero piegandosi al peso che l'avversario, cugino della duchessa Bianca Maria Visconti, aveva a corte e a livello locale ${ }^{13}$.

Nonostante fosse incoraggiata la buona volontà degli individui e della collettività di rinunciare ai cavilli frivoli del diritto positivo (le exceptiones per eccellenza nelle nostre fonti pragmatiche) il signore della deroga era senzaltro il principe. Egli poteva mitigare la norma avendo speciale considerazione della singolarità dei luoghi e dei tempi, senza tralasciare qualche riguardo per gli stessi sudditi. Antonio Mattia da Iseo apparteneva a quel fronte ghibellino che, con la conquista veneziana dei territori di Bergamo e Brescia, aveva scelto di espatriare, lasciando beni e giurisdizioni per accettare più umili ruoli

\footnotetext{
12 Gentile, Fazioni al governo, p. 200 per le citazioni. Su Antonio e questa parentela di ufficiali, si veda Roveda, I Colli di Vigevano.

13 Covini, Potere, ricchezza, p. 188 per le citazioni. Nella stessa scrittura ricorre anche nientedimanco, in un passaggio di minore interesse.
} 
funzionariali al servizio dei Visconti e degli Sforza. Da podestà di Morbegno si trovò a reggere una giurisdizione che descrisse a Francesco Sforza come violenta e indocile. Fra l'altro era necessario che «divisioni et partialità» locali, sempre aborrite, fossero estirpate, «ma nondimeno che la provisione fusse temperata havendo rispecto alle confine et per non mostrare che la signoria vostra se defidi però de questo luoco», cioè considerando la posizione di frontiera con lo stato veneto e l'opportunità di non indispettire o avvilire la popolazione ${ }^{14}$.

Il podestà di Bormio Francesco Creppa, incaricato dell'inchiesta, sapeva che i nobili del Tiranese non volevano che la giurisdizione fosse affidata a Mario Federici. Anche se non sancito ufficialmente, quest'ordine oligarchico del paese era così radicato nella prassi dello stato regionale che la trama di relazioni concordi fra lui, rappresentante del duca, e questi «gentilhomini» che «sono prencipio, mezo et fine de dicta iurisdictione», gli sembrava sufficientemente integra da non richiedere ulteriori consultazioni, estese agli altri ceti. «Nondimeno, piacendo a vostra excelentia proceda più ultra, per tore la voce cossì populare como de gentilhomini, sono aparechiato ${ }^{15}$.

Questa sensibilità per il carattere variegato delle circostanze, propria del duca e che sotto la sua autorità avrebbe dovuto esprimere anche chi lo rappresentava in periferia, si poneva però in potenziale conflitto con la suscettibilità dei corpi territoriali, per i quali, come era già chiaro a Chabod, la puntigliosa conservazione di consuetudini, statuti e privilegi era lo strumento di salvaguardia delle autonomie e delle istituzioni di ascendenza comunale ${ }^{16}$. Cominciamo quindi dalla consuetudine. Una lettera scritta alla reggente Bona di Savoia e a Gian Galeazzo Maria Sforza nel 1479 da Carlo Favagrossa da Cremona, che ebbe fra l'altro l'importante incarico centrale di maestro delle entrate ordinarie e in quel momento era podestà e commissario di Bellinzona, oppose il dato del mutare dei tempi e della stessa natura al rispetto della tradizione, cui la comunità si appoggiava e che l'ufficiale lasciava cadere con insolita nettezza di fronte all'incalzare dell'adesso. L'eccezione all'usitato dunque qui è già nelle cose, la congiunzione nondimanco introduce l'impegno del valido ufficiale nella ricerca degli ulteriori accomodamenti a questa realtà variabile e contraddittoria. Era gennaio e i soldati alloggiati fra quelle montagne avevano bisogno di procurarsi la legna, evidentemente per riscaldarsi oltre che per eventuali manufatti necessari agli stanziamenti. Per gli «homini de la terra» essi dovevano rifornirsi nei boschi comuni, «como se solea fare al tempo de lo illustrissimo duca Filippo \&c.», abbreviava lo scrivente spazientito dal passatismo degli abitanti. I boschi comuni, però, presumibilmente perché già intaccati dai cantieri fortificatori che via via lungo il secolo furono attivi presso il borgo e spogliati dal commercio che alimentava il fabbisogno di le-

14 Archivio di Stato di Milano [ASMi], Carteggio sforzesco [CS], 720, 1463.01.12.

15 ASMi, Comuni, 12, Bormio, 1477.08.13.

16 Signori, regimi signorili. 
gname di Milano, si erano ritirati lontano dall'abitato ed era diventato faticoso servirsene. «Aviso le illustrissime signorie vostre che alhora era uno tempo et adeso un altro, perché li boschi erano su le porte de Belinzona et adesso sonno lontani quatro et cinque milia, onde li pare assay difficile. Niente de manco (...) ne sforzarò de adaptare» ${ }^{17}$.

Più arditamente, il comando del principe e la conseguente azione dell'ufficiale poteva sfidare non solo il solitum, ma anche ciò che veniva considerato oggettivamente giusto (il debitum). Nel 1452 Francesco Sforza manifestò il suo disappunto al commissario di Como Tommaso Tebaldi da Bologna nella forma della meraviglia per non avere egli atteso ad un ordine, quello di far eseguire le impegnative garanzie pecuniarie («segurtade») prestate da Pietro da Binago e Giovanni da Castiglione e di fare arrestare Maffeo da Como, «muratore», presumibilmente un loro collega. Il destinatario, uomo colto e figura di peso dell'ufficialità visconteo-sforzesca, che esercitò anche incarichi diplomatici e di organizzazione militare, divenendo membro del più importante organismo dello stato, il Consiglio segreto, dichiarava che «da puto io imparay a hobedire et cussi sempre farò», però sentiva una viva contraddizione. Riteneva che i diritti vantati contro i primi due «magistri» non sussistessero e che Maffeo fosse un «bon zovene», per il quale invocava la «compassione» del duca. «Nondimeno, se la excelentia vostra vole ch'io le astrenza, o debito o indebito che sia, farò contro loro e contra ogni altro tuto quello che la excelentia vostra me comandarà», eccezionale espressione di una disponibilità senza reticenze a seguire gli ordini anche contro il buon diritto ${ }^{18}$.

È evidente a questo punto che nel rapporto diretto fra l'autorità centrale e i corpi territoriali, l'eccezione vada a demarcare l'autorità che il principe si attribuisce di non rispettare la norma e di agire nonostante (altra congiunzione molto pregnante) gli impegni presi. Francesco Sforza scrisse agli uomini di Teglio per ammettere che «dessemo speranze» ai loro messi di confermare il podestà in carica; «nondimeno» si era alla fine stabilito in modo diverso, destinando all'ufficio un'altra persona, «siché volemo lo acceptate», però con la rassicurazione che le usuali garanzie non venivano meno («non vi lasseremo fare iniuticie né mali tractamenti») ${ }^{19}$.

Così dal principe a chi gli era più vicino fino $\mathrm{a}$ chi lo rappresentava nelle periferie si estendeva la possibilità di fare perlomeno temporaneamente eccezione alla legalità. Anche un osservatore esterno meditava su questa con-

\footnotetext{
${ }^{17}$ Ticino ducale, III/3, p. 69, doc. 1363. In una lettera inviata da Domodossola, di tono però molto più moderato, Ambrogino da Longhignana, capo militare che fece una brillante carriera, culminata nel comando della guarnigione del castello di Porta Giovia a Milano, informò il duca e la duchessa dell'ufficio del contrascriptore dei lavori di fortificazione del borgo, del suo salario e della pratica di destinarvi un abitante del luogo; «niente di meno» suggerì l'infrazione del precedente per evitare conflitti d'interesse (ASMi, Autografi, 83, fasc. 63, 1479.01.13). Su queste figure, si veda anche Santoro, Gli uffici del dominio sforzesco.

${ }_{18}$ Motta, Architetti e ingegneri, p. 142. Sul Tebaldi, si veda, fra gli altri, Margaroli, Diplomazia e stati, ad indicem; Il ducato di Filippo Maria Visconti.

19 ASMi, CS, 1622, 1465.05.11.
} 
dizione, le sue opportunità e i suoi limiti, esprimendo la solita sensibilità per i distinguo e la stima di questa età per il senso delle cose maturato empiricamente. L'oratore mantovano Zaccaria Saggi nel 1477 riferì che senz'altro Cicco Simonetta di fatto governava con uno stile irrituale e poco gradito («haveria potuto servare altri modi et più accepti a la brigata che'l non ha, et non doveva voler dimostrare superiorità fra gli altri come l'ha fatto»); «nientedimeno, chi considera bene el bisogno di questo stato, meser Cecho gli è più che necessario per la grande et longa experientia che'l ha nel suo exercitio» ${ }^{20}$.

Anche i sudditi usano la delicata congiunzione, ma proprio per prevenire il rischio di possibili scostamenti dagli attributi tradizionali della sovranità come tutrice dei diritti. Il comune di Tirano temeva che il duca potesse rinunciare a difenderne il contestato possesso dei pascoli di montagna situati lungo il confine del dominio contro gli agguerriti vicini, gli uomini di Poschiavo e Brusio, per non inimicarsi il loro signore, il vescovo di Coira: «benché conoschamo con effeto (...) che quella [signoria] non ne volle amanchare de raxone, nondimancho ne pare per la presente di novo ricomandarse et pregiamo se voglia ricordarse de questa povera comunitate» ${ }^{21}$.

Soprattutto la messa in questione dei capitoli di dedizione, la base pattizia della ricostruzione del dominio da parte di Francesco Sforza, poteva essere la premessa di uno scontro istituzionale ${ }^{22}$. Insolitamente battagliera è la supplica della corte di Mattarella (Ossola superiore), in cui sia la continuità della consuetudine, sia la limitazione convenzionale del potere sovrano mediante i capitoli stridevano con l'innovazione decisionale. I Visconti non avevano mai imposto alla comunità la tassa del sale, gli Sforza avevano confermato lo status quo «fin al presente (...) servato. Nondimeno, contra lo usitato sempre may et contra la dispositione di essi capituli», si era disposto altrimenti, sicché i sudditi si mostravano increduli, "attenduta la inveterata consuetudine et capituli, sì perché la signoria vostra già non è usata de inovare a li boni stilli et usanze servati continue al tempo de li prefati signori Vesconti $»^{23}$.

Il comune di Teglio sviluppava la stessa sostanza argomentativa, con toni più morbidi e proponendo in modo meno aspro il motivo di tensione fra l'eccezione principesca e la norma difesa dalla repubblica ${ }^{24}$. La terra era dotata di propri statuti e di un proprio podestà, ma gli uditori di Ascanio Sforza ne avevano violato la giurisdizione, rinviando una causa al capitano di Valtellina «cum clausola 'privilegiis non attentis'». Gli uomini volevano preservare una diversa immagine del duca, ricorrendo, con un uso ancora eccezionale

\footnotetext{
${ }^{20}$ Covini, Potere, ricchezza, p. 231, nota 4 per la citazione (dove si inquadrano le testimonianze del Carteggio degli oratori mantovani, $\mathrm{X})$.

21 ASMi, CS, 1156, 1493.10.31.

22 Chittolini, Città, comunità.

23 ASMi, Comuni, 42, Matarella, s.d. Meno polemicamente il borgo di Varese affermò che la «usata» misura della tassa del sale istituita da Filippo Maria Visconti dovesse valere anche con i successori; «nondimeno» quella gravezza era stata appesantita (ASMi, Comuni, 85, Varese, s.d.).

24 Nel senso ricostruito da De Benedictis, Repubblica per contratto.
} 
in queste scritture, al concetto di natura per limitarne gli attributi alle azioni tradizionali: onorare la propria fede, che ricambia specularmente la fede dei sudditi, osservare le immunità, non infrangere il precedente. «Tanto più sinceramente ricoremo a la prelibata excelentia vostra quanto che naturalmente quella è inclinata a servare la fede et ogni opera de iustitia». Ovviamente non c'era neanche bisogno di richiamare il principe alla custodia del bene comune e alla conservazione delle immunità riconosciute, tuttavia un prudente ricordo non avrebbe guastato («benché cognoscamo non essere necessario persuadere la excelentia vostra a la observatione di nostri privilegii in farla a la com[m]une utilitate di la sua et nostra republica di la castelantia di Telio [...], nondimeno vogliamo pregare quella a la iusticia et observatione di quilli») ${ }^{25}$.

Diverse mi paiono le dinamiche dei rapporti fra le comunità stesse e gli uffici territoriali. È vero che tendenze al comportamento extra-legale erano diffuse fra gli stessi rappresentanti periferici del principe, frequentemente accusati di esosità e prepotenza, ma la questione era più generale. Nello stato tardo-medievale, in assenza di un diritto amministrativo di tipo moderno, era continua la competizione fra diversi attori istituzionali che si disputavano prerogative e pretendevano competenze giurisdizionali sulla base di opposti precedenti. Più che una vera e propria protesta di portata costituzionale, come nei casi precedenti, qui legalità e torto sono senz'altro posti in contraddizione, e con minori reticenze di quelle impiegate per rivolgersi al principe, ma essenzialmente con lo scopo di rivendicare esenzioni o prerogative in una realtà istituzionale definita in modo estremamente incerto e caduco. In una supplica del comune lariano di Mandello si respingeva così ogni dovere di concorrere alle spese per la manutenzione di una fortificazione di confine in Valsassina: «benché li vostri (...) subditi (...) non siano obligati in cossa alchuna verso el castelano de la rocha de Bayedo, vestre Vallissaxine, nondimeno epso castelano ad dì passati ha fatto fare alchuni sequestri de' beni de alchuni d'epsi homini $»^{26}$. A proposito dello stesso problema, nel 1500 protestava il comune di Bellano. «Licet del anno 1494 la vostra signoria, per sue lettere patente (...) liberasse la vostra fidelissima comunitate (...), nondimanco in l'anno passato» il luogotenente del podestà della Valsassina, Sozzino Arrigoni, «contra ogni debito de raxone», aveva disposto un sequestro di animali da macello. «Et quantoncha li fosse fato intendere che la dicta comunitate era exempta di tal caricho (...), nondimanco esso Sozino, como presumptuoso, (...) may ha voluto restituire esse bestie ${ }^{27}$.

Nel carteggio cinquecentesco la congiunzione torna in contesti analoghi, testimoniando la profondità e la durata di questa cultura. Concludo pertanto con una sola citazione, che ci proietta al fuori del dominio milanese. Giovanni Morone, proveniente da una famiglia che aveva scalato la piramide degli uffici sforzeschi (il padre Gerolamo occupò in sostanza il ruolo che fu del Si-

25 ASMi, CS, 1157, 1498.02.18.

26 ASMi, Comuni, 5, Baiedo, s.d.

27 ASMi, Comuni, 5, Baiedo, 1500.04.02. 
monetta) e allevato nel grembo di quell'esercizio accorto del potere, giurista di formazione come gli avi, divenuto cardinale si trovò a fronteggiare, con la sua propensione alla mediazione, le nuove intransigenze del conflitto confessionale. Diplomatico stimatissimo, attento alla «diversità de' tempi» e alla «mutatione» che dunque si impone, a «quello che si conviene» nelle diverse situazioni, fu incaricato da Gregorio XIII anche del compito squisitamente politico della pacificazione di Genova (1575-1576). Verso la fine del suo incarico, scrisse che avrebbe voluto escludere dallo status nobiliare, di cui si cercava una nuova definizione, gli operatori di gabelle, dazi e cambi, ma aveva dovuto ripiegare su un compromesso. Lo scopo sarebbe stato «rimovere a fatto questo mal uso et tanto perverso dell'usure (...), nondimeno tutto si è accomodato (...) per pigliare il bene non possendo havere il meglio» ${ }^{28}$.

\section{I lenti e controversi progressi dell'eccezione}

I tormentati passaggi proposti evidenziano dilemmi scoperti nella politica tardo-medievale e proto-moderna. Ginzburg riporta nella prima pagina del suo libro la celebre definizione di Carl Schmitt, «sovrano è chi decide sullo stato di eccezione», che sintetizza efficacemente un insieme di categorie che hanno stimolato la riflessione su questi temi negli ultimi decenni. Si deve d'altra parte constatare che proprio nel periodo considerato la funzione monarchica sta ancora molto faticosamente forzando il perimetro dei suoi attributi tradizionali. È un aspetto su cui insiste una delle revisioni critiche della statualità che hanno avuto maggiore impatto storiografico nel Novecento, quella di Otto Brunner. È singolare, proprio a proposito dei problemi qui affrontati, che a partire da elementi parziali della sua interpretazione siano state tanto enfatizzate le convergenze con Schmitt ${ }^{29}$. È una lettura che sconta l'appiattimento, su una generica Weltanschauung ideologica, di un campo di culture politiche ben più frastagliato. Una contestualizzazione più fine, infatti, colloca Brunner, pure senz'altro portato a enfatizzare le funzioni di dominio, ben lontano dalle politologie del cesarismo, formatosi piuttosto nel rapporto (comunque critico) con il tradizionalismo cattolico austriaco ed entro l'ampia corrente della rivoluzione conservatrice e dei suoi tipici motivi di contestazione della modernità borghese e del suo universo dicotomico (la separazione e il predominio acquisito dall'economia sulla politica, l'appiattimento delle relazioni sociali nelle masse urbane, il livellamento dei multiformi gruppi corporati dell'antico regime nell'individualismo, le geometrie astratte dello stato e del mercato che hanno dissolto gli spazi custoditi e "concreti" della casa e del territorio) ${ }^{30}$. La costellazione costituita da sovranità-decisione-eccezione, che vuole proietta-

\footnotetext{
${ }^{28}$ Firpo, Maifreda, L'eretico che salvò la Chiesa, specialmente pp. XV e sgg., 570, 772, 825 per le citazioni.

29 Algazi, Herrengewalt und Gewalt.

$3^{0}$ Van Horn Melton, From Folk History.
} 
re la leadership al di là della legalità, risulta pertanto radicalmente estranea ai paesaggi brunneriani del potere. «La sovranità del sovrano comincia ad instaurarsi solo nel momento in cui quest'ultimo (...) è nella condizione di poter effettivamente decidere per proprio conto. Ma tutto questo non avrà luogo che a partire dal XVI secolo» (p. 559). Al di là delle citazioni reciproche, le stesse tangenze fra i due autori paiono piuttosto estrinseche, quasi, per così dire, dei falsi amici. Il concetto di costituzione materiale è impugnato in modo diverso: Schmitt è alla ricerca di un ordine giuridico eventualmente contra legem, al contrario a Brunner preme affermare la vincolante saldezza del «fondamento giuridico» delle relazioni territoriali, non dipendente in modo esclusivo dal sovrano, «pur mancando del sostegno di una carta costituzionale» (p. 596). La forza non costituisce per Brunner un elemento di rottura della legge, ma un esercizio giuridicamente riconosciuto che mantiene attivo il proprio buon diritto. Ancora, la polemica con Hans Kelsen e il positivismo giuridico presenta ragioni pressoché rovesciate: Schmitt respinge la riduzione dell'espressione del politico alla legalità, Brunner la riduzione del diritto al solo diritto dello stato. È conseguente che in questo quadro, in cui il diritto non ha una fonte solo statuale e non è nella disponibilità del monarca, che piuttosto lo garantisce in vista del «bene comune», cioè della pace (p. 560), senza detenere il monopolio della forza di renderlo esecutivo e tantomeno di oltrepassarlo, per l'eccezione o l'emergenza non si manifesti nessun interesse analitico, a vantaggio della regolarità della consuetudine che sovrasta tanto il popolo quanto i signori. «Nella visione giuridica del medioevo non c’è possibilità alcuna per il signore di oltrepassare arbitrariamente i confini prescritti al suo operato» e una violazione renderebbe legittima la resistenza (p. 617) ${ }^{31}$.

Eppure il processo che Brunner vuole situare, almeno nel suo compimento e nella realtà regionale che studia, nella piena o tarda età moderna, fra XIV e XV secolo era già in corso, moltiplicando proprio attorno alla legge e alle sue eccezioni generalizzati motivi di attrito politico. Essi affiorano dai trattati celebri come dalle suppliche delle comunità rurali, con assonanze da non sottovalutare e da non interpretare unilateralmente, astraendone un canone intellettuale limitato a poche voci "originali". Ampliando la gamma di pratiche già in uso in età comunale, ma infrangendo le salvaguardie formali proprie della cultura duecentesca delle istituzioni, repubbliche e principati italiani istituirono cariche individuali rivestite di poteri speciali o organismi con funzioni d'emergenza. La giustizia, quale era regolata dagli statuti, fu messa sotto pressione dalle procedure sommarie e segrete o per contro dall'uso massiccio della grazia principesca, alimentata dalla richiesta di un addolcimento equitativo del rigor iuris proveniente dagli stessi sudditi ${ }^{32}$. Un approdo ancora

\footnotetext{
${ }^{31}$ Brunner, Terra e potere. Si veda, anche come sintesi di un amplissimo dibattito, Consolati, Dominare tempi inquieti.

${ }^{2}$ Cozzi, Repubblica di Venezia; Suppliche e «gravamina»; Tanzini, Il governo delle leggi; Sistemi di eccezione; Tecniche di potere, oltre all'imponente lavoro confluito in Italia comunale e signorile.
} 
più generale potrebbe essere considerato lo sbrigativo pragmatismo con cui si voleva fosse eseguita la volontà del monarca, perché in fondo c'è eccezione laddove c'è comunque regola, mentre qui si trattava di una duttilità del comando e di una altrettanto plastica obbedienza come risposte pragmatiche e flessibili alle contingenze.

Per restare all'interno del perimetro dello stato di Milano, già i Visconti si erano riservati di derogare alla legge positiva, la pressione sullo ius proprium si era intensificata, con i successori di Francesco Sforza si erano dilatati gli interventi di giustizia e di governo discrezionali, si erano cercati nelle periferie interlocutori politici diversi dalle istituzioni formalizzate, a vantaggio di più nebulosi gruppi di principali33 ${ }^{3}$. Negli stessi capitoli che davano al rapporto di dominio una veste di convenzione bilaterale poteva trovare spazio il caso di necessità. Pedullà rileva che «il termine tecnico per riferirsi a questa condizione eccezionale che giustificava il ricorso a rimedi altrettanto eccezionali era necessitas», appartenente ad una lunga tradizione antica e medievale, voce viva del dibattito quattrocentesco e di cui Machiavelli si serve per una pronunciata espansione dell'autorità del principe ${ }^{34}$. Ricordo incidentalmente che il «necessitatis casus» è valorizzato anche da Schmitt ${ }^{35}$. Ebbene, al comune di Bormio che chiedeva che gli uomini non potessero essere convocati alle armi al di là dei confini del territorio, anteponendo la necessità di difendere le loro case («cum eis domi pro defensa peropportuni et necessarii sint»), Francesco Sforza concedeva un assenso che però già implicava l'eccezione di una ben più astratta necessità («acceptamus, nisi in casu necessitatis») ${ }^{36}$.

Insomma, adattare il quadro normativo e istituzionale all'«esigenza di circostanze, tempi, persone» è un grande tema della politica del tempo, direi più ampio e prioritario rispetto a quello del principe come signore dell'eccezione, che una visuale ex post può condurre a sopravvalutare nella sua portata. Anzi, nei Discorsi è la repubblica ritenuta più idonea ad «acccomodarsi alla diversità de' temporali». D'altra parte è vero che si stava affermando una diversa risposta: il pensiero, che Schmitt sintetizzerà con le citate parole di Jean Bodin, secondo cui è invece l'azione extra-legale del sovrano la più efficace nel contemplare la peculiarità e nel controllare l'imprevisto, peraltro mediante apparati sempre meno improvvisati ${ }^{37}$.

$\mathrm{Si}$ tratta, evidentemente, di processi di lungo periodo, di cui è bene non forzare letture anacronistiche. La cultura consuetudinaria e il tradizionale

\footnotetext{
33 Covini, «La balanza drita», pp. 259-328; Storti Storchi, Scritti sugli statuti; Cengarle, Lesa maestà, pp. 73-80; Gamberini, La legittimità contesa; Del Tredici, Il partito dello stato.

34 Pedullà, Machiavelli secondo Carlo Ginzburg, pp. 22-25.

35 Schmitt, Le categorie del 'politico', p. 37.

${ }^{36}$ ASMi, $C S, 1522,1450.03 .23$.

37 Il passaggio machiavelliano è inquadrato da Ginzburg, Nondimanco, pp. 43-61. La frase di Bodin è riportata da Schmitt, Le categorie del 'politico', p. 36. Per approfondire la connessione, nel discorso politico quattrocentesco, fra «la variabilità imprevedibile dei casi umani, che la legge (...) non può comprendere» e la "giustificazione etico-giuridica dei modi di governo 'extraordinarii'», rinvio ancora a Fubini, Italia quattrocentesca, pp. 172, 174 per le citazioni.
} 
legalismo godettero senz'altro di grande credito per tutto il Quattrocento e oltre; l'intangibilità dei diritti riconosciuti costituì la persistente bandiera politica dei corpi territoriali e dei signori locali ${ }^{38}$. Nondimeno si venivano a moltiplicare gli impulsi dei principi, anche degli Sforza, volti ad aprire delle brecce nell'edificio consuetudinario che permettessero loro di farsi arbitri della circostanza, contenuti dai sudditi che non mancarono, all'occorrenza, di richiamare i duchi, e chi li rappresentava nelle periferie, a servare senza eccezioni gli impegni assunti e la norma approvata.

${ }^{38}$ Arcangeli, Piccoli signori. 


\section{Opere citate}

G. Algazi, Herrengewalt und Gewalt der Herren im späten Mittelalter. Herrschaft, Gegenseitigkeit und Sprachgebrauch, Frankfurt-New York 1996.

L. Arcangeli, Piccoli signori lombardi e potenze e grosse, in Linguaggi politici nell'Italia del Rinascimento, a cura di A. Gamberini e G. Petralia, Roma 2007.

C. Baja Guarienti, Il bandito e il governatore. Domenico d'Amorotto e Francesco Guicciardini nell'età delle guerre d'Italia, Roma 2014.

O. Brunner, Terra e potere. Strutture pre-statuali e pre-moderne nella storia costituzionale dell'Austria medievale, Milano 1983 (Wien 1965).

The Cambridge Companion to Machiavelli, a cura di J.M. Najemy, Cambridge 2010.

Carteggio degli oratori mantovani alla corte sforzesca, X, 1475-1477, a cura di G. Battioni, Roma 2008.

F. Cengarle, Lesa maestà all'ombra del biscione. Dalle città lombarde ad una 'monarchia' europea (1335-1447), Roma 2014.

F. Chabod, La genesi del «Principe» e l'esperienza delle cose d'Italia, in La crisi degli ordinamenti comunali e le origini dello stato del Rinascimento, a cura di G. Chittolini, Bologna 1979, pp. 323-342.

G. Chittolini, Città, comunità e feudi negli stati dell'Italia centro-settentrionale (secoli XIV-XVI), Milano 1996.

W.J. Connell, Machiavelli nel Rinascimento italiano, Milano 2015.

I. Consolati, Dominare tempi inquieti. Storia costituzionale, politica e tradizione europea in Otto Brunner, Bologna 2020.

N. Covini, «La balanza drita». Pratiche di governo, leggi e ordinamenti nel ducato sforzesco, Milano 2007.

N. Covini, Potere, ricchezza e distinzione a Milano nel Quattrocento. Nuove ricerche su Cicco Simonetta, Milano 2018.

G. Cozzi, Repubblica di Venezia e stati italiani. Politica e giustizia dal secolo XVI al secolo XVIII, Torino 1982.

A. De Benedictis, Repubblica per contratto. Bologna: una città europea nello stato della Chiesa, Bologna 1995.

F. Del Tredici, Il partito dello stato. Crisi e ricostruzione del ducato visconteo nelle vicende di Milano e del suo contado (1402-1417), in Il ducato di Filippo Maria Visconti, 1412-1447. Economia, politica, cultura, a cura di F. Cengarle e M.N. Covini, Firenze 2015, pp. 27-69.

Il ducato di Filippo Maria Visconti, 1412-1447. Economia, politica, cultura, a cura di F. Cengarle e M.N. Covini, Firenze 2015.

E. Fasano Guarini, Repubbliche e principi. Istituzioni e pratiche di potere nella Toscana granducale del '50o-'60o, Bologna 2010.

M. Firpo, G. Maifreda, L'eretico che salvò la Chiesa. Il cardinale Giovanni Morone e le origini della Controriforma, Torino 2019.

R. Fubini, Italia quattrocentesca. Politica e diplomazia nell'età di Lorenzo il Magnifico, Milano 1994.

R. Fubini, Politica e pensiero politico nell'Italia del Rinascimento. Dallo stato territoriale al Machiavelli, Firenze 2009.

A. Gamberini, La legittimità contesa. Costruzione statale e culture politiche (Lombardia, XII-XV sec.), Roma 2016.

M. Gentile, Fazioni al governo. Politica e società a Parma nel Quattrocento, Roma 2009.

C. Ginzburg, Nondimanco. Machiavelli, Pascal, Milano 2018.

C. Ginzburg, Gabriele Pedullà e la carta della Cina, in «Storica», 24 (2018), 72, pp. 193-196.

Italia comunale e signorile, collana diretta da J.-C. Maire Vigueur e A. Zorzi, Roma 2013-

I. Lazzarini, Argument and emotion in Italian diplomacy in the early fifteenth Century: the case of Rinaldo degli Albizzi (Florence, 1399-1430), in The languages of the political society, a cura di A. Gamberini, J.-Ph. Genet e A. Zorzi, Roma 2011, pp. 339-364.

S. Leprai, Il governo del disordine ai confini di uno stato. Borgotaro e gli Sforza (1467-1488), Bologna 2011.

P. Margaroli, Diplomazia e stati rinascimentali. Le ambascerie sforzesche fino alla conclusione della Lega italica (1450-1455), Firenze 1992.

E. Motta, Architetti e ingegneri militari sforzeschi (repertorio di fonti e notizie sommarie), in «Bollettino storico della Svizzera italiana», 12 (1890), pp. 141-144. 


\section{Massimo Della Misericordia}

G. Pedullà, Machiavelli secondo Carlo Ginzburg, in «Storica», 24 (2018), 71, pp. 9-86.

D. Quaglioni, Machiavelli e la lingua della giurisprudenza. Una letteratura della crisi, Bologna 2011.

E. Roveda, I Colli di Vigevano: una famiglia lombarda nelle strutture di governo dello stato (secoli XIV-XVI), di futura pubblicazione.

C. Santoro, Gli uffici del dominio sforzesco (1450-150o), Milano 1948.

C. Schmitt, Le categorie del 'politico'. Saggi di teoria politica, Bologna 1972.

Signori, regimi signorili e statuti nel tardo medioevo, a cura di R. Dondarini, G.M. Varanini e M. Venticelli, Bologna 2003.

Sistemi di eccezione, a cura di M. Vallerani, in «Quaderni storici», 44 (2007), 2, pp. 299-547.

La Storia d'Italia di Guicciardini e la sua fortuna, a cura di C. Berra e A.M. Cabrini, Milano 2012.

C. Storti Storchi, Scritti sugli statuti lombardi, Milano 2007.

Suppliche e «gravamina». Politica, amministrazione, giustizia in Europa (secoli XIV-XVIII), a cura di C. Nubola e A. Würgler, Bologna 2002.

L. Tanzini, Il governo delle leggi. Norme e pratiche delle istituzioni a Firenze dalla fine del Duecento all'inizio del Quattrocento, Firenze 2007.

Tecniche di potere nel tardo medioevo. Regimi comunali e signorie in Italia, a cura di M. Vallerani, Roma 2010.

Ticino ducale. Il carteggio e gli atti ufficiali, III/3, Gian Galeazzo Maria Sforza. Reggenza di Bona di Savoia. 1479-148o, a cura di G. Chiesi, Bellinzona 2014.

N. Tranchedini, Vocabolario italiano-latino. Edizione del primo lessico dal volgare. Secolo XV, a cura di F. Pelle, Firenze 2001.

J. Van Horn Melton, From Folk History to Structural History: Otto Brunner (1898-1982) and the Radical Conservative Roots of German Social History, in Paths of Continuity. Central European Historiography from the 1930 s to the 1950s, a cura di H. Lehmann e J. Van Horn Melton, Cambridge 1994, pp. 263-292.

Massimo Della Misericordia

Università degli Studi di Milano Bicocca

massimo.dellamisericordia@unimib.it 